Ciência e Natura, Santa Maria, v. 37 n. 4 set-dez. 2015, p. 684-692

Revista do Centro de Ciências Naturais e Exatas - UFSM

ISSN impressa: 0100-8307 ISSN on-line: 2179-460X

\title{
ciênciaenatura
}

\section{Produção de documentário para o ensino da coleta de sangue venoso}

\author{
Production of a documentary for the teaching of venous blood collection
}

\author{
Marciano Robson de Mirandaㄹ, Camilo Amaro de Carvalho², Vitor Marcelino Mendes Martins de \\ Carvalho ${ }^{1+I n}$ memore, Paula Sannazzaro Oliveira de Assis ${ }^{1}$, Valéria Cristina de Faria ${ }^{3}$, Luciana Moreira \\ Lima $^{4}$
}

${ }^{1}$ Acadêmicos em Medicina, Universidade Federal de Viçosa- UFV, Brasil. ${ }^{2}$ Farmacêutico, Doutor em Biologia Celular e Estrutural, Departamento de Medicina e Enfermagem - UFV, Viçosa, Brasil. ${ }^{3}$ Educadora física. Mestre em Educação Física, Educadora física do Agros - Instituto UFV de Seguridade Social, Viçosa, Brasil.

${ }^{4}$ Farmacêutica, Doutora em Ciências Farmacêuticas, Departamento de Medicina e Enfermagem - UFV, Viçosa, Brasil.

\section{Resumo}

A medicina laboratorial é uma especialidade responsável pela realização de exames complementares à prática clínica médica, sendo a coleta de sangue venoso uma das técnicas mais utilizadas nos serviços de saúde. O ensino desta subárea da medicina, como a educação médica de modo geral, vem sendo reformulado de modo a incluir novas metodologias, uma delas o uso de materiais audiovisuais. O estudo teve por objetivo desenvolver um documentário para o ensino de coleta de sangue venoso, buscando demonstrar todos os passos desse procedimento, bem como detalhar outras variáveis pré-analíticas que podem causar interferências nos exames laboratoriais. Para a confecção e a validação do documentário foram obtidas mais de 60 minutos de filmagem, editados para um documentário de 18 minutos. Para validação foram utilizados três diferentes instrumentos: portfólios, grupos-focais aplicados aos autores e questionários aplicados em diferentes públicos. Após aplicação dos questionários, foi constatado que os recursos audiovisuais funcionam como um bom método de ensino, complementar ao ensino tradicional. Este estudo não postula, porém, a superioridade de um método em relação ao outro, apenas afirma que o uso de documentários mostrou-se como um eficaz e importante instrumento didático para auxílio no ensino.

Palavras-chave: Ensino, Coleta de amostras de sangue, Educação Continuada, Documentário.

\begin{abstract}
Introduction: The Laboratory Medicine is the medical specialty responsible for conducting tests which complement the practice of medical clinics and the venous blood collection is one of the techniques most commonly used in health services. The teaching of this sub-area of medicine, such as medical education in general, has been redesigned to include new approaches, one of them being the use of audiovisual materials. Objectives: This paper reports the confection and the validation process of a documentary developed by the authors for the teaching venous blood collection. Method: The recordings were made in collaboration with the Coordenadoria de Educação Aberta e a Distância (CEAD/UFV) and the Laboratório de Análises Clinicas da Divisão de Saúde da UFV (LAC/DSA). Over 60 minutes of footage were obtained, edited into a documentary of 18 minutes. For the validation three different instruments were used: portfolios and focus groups applied to authors and questionnaires applied to certain publics. Results: After applying the questionnaires we found that audiovisual resources serve as a good teaching method, complementary to traditional teaching. Conclusions: This study does not postulate the superiority of one method over the other, merely states that the use of documentaries was shown as an effective and important educational
\end{abstract} tool.

Keywords: Teaching, Blood Collection, Education Continuing, Documentary. 


\section{Introdução}

A medicina laboratorial é uma especialidade voltada para a realização de exames complementares para auxiliar na prevenção, diagnóstico, prognóstico e acompanhamento terapêutico. ${ }^{1}$ A realização de exames laboratoriais faz parte do processo de diagnóstico de diversas patologias, que apenas são detectadas através de análise das amostras biológicas. Nos laboratórios de análises clínicas, as análises são fundamentadas em um processo dinâmico que se inicia na coleta do espécime diagnóstico (amostra biológica obtida adequadamente para fins de diagnóstico laboratorial) e termina com a emissão de um laudo. Didaticamente, o processo pode ser dividido em três fases: pré-analítica, analítica e pós-analítica. ${ }^{2}$

Entre os exames realizados no laboratório clínico, a coleta de sangue venoso é um dos procedimentos mais realizados nos serviços de saúde, o que ressalta a importância de uma boa técnica de coleta, pois esta é essencial para a confiabilidade dos resultados laboratoriais.

Segundo as recomendações Sociedade Brasileira de Patologia Clínica/Medicina Laboratorial (SBPC/ML) ${ }^{2}$ para coleta de sangue venoso, a fase pré-analítica é responsável por $70 \%$ do total de erros que ocorrem em um laboratório clínico. A fase pré-analítica, que inclui a indicação do exame, a redação da solicitação, instruções de preparo ao paciente, avaliação do atendimento às condições prévias, procedimentos de coleta, acondicionamento, preservação e transporte da amostra biológica até a realização efetiva do exame, é apontada por ser responsável por cerca de $70 \%$ do total de erros ocorridos nos laboratórios clínicos. ${ }^{2,3}$

Diante da relevância desse conteúdo, o ensino da disciplina medicina laboratorial, especificamente o ensino de coleta de sangue venoso, aos acadêmicos de medicina, instiga o surgimento de novas metodologias que facilitem o processo de ensino-aprendizagem do estudante.
Nessa perspectiva, os documentários são utilizados como recursos audiovisuais, que quando desenvolvidos com um roteiro e linguagem apropriados para o público-alvo, atuam como elementos geradores ou mesmo fixadores de conteúdo, pois permitem que o aluno possa compreender os conteúdos propostos de forma lúdica, facilitando o aprendizado para aqueles que não conseguem abstrair com tanta facilidade. Esta metodologia facilita o processo ensino-aprendizagem do estudante, combatendo a monotonia de aulas excessivamente conteudistas e pouco contextualizadas ${ }^{4,5}$, além de possibilitar abranger um número maior de pessoas e fornecer informações padronizadas. ${ }^{6}$

Dessa forma, tais instruções fornecidas aos acadêmicos de medicina, por meio de um documentário, possibilitam o contato contínuo com um material de qualidade, atuando como um instrumento educativo para o aluno, assim como um objeto lúdico de divulgação que estimula a criatividade e o interesse desses. ${ }^{7}$

Portanto, o objetivo do presente estudo foi desenvolver um documentário para o ensino de coleta de sangue venoso, buscando demonstrar todos os passos desse procedimento, bem como detalhar outras variáveis préanalíticas que podem causar interferências nos exames laboratoriais.

\section{Material e métodos}

O projeto foi aprovado pelo Comitê de Ética em Pesquisa com Seres Humanos da Universidade Federal de Viçosa (UFV) e todos participantes assinaram o Termo de Consentimento Livre e Esclarecido e a autorização para veiculação de sua imagem no DVD. Firmou-se uma parceria com a Coordenadoria de Educação Aberta e a Distância (CEAD/UFV), sendo a equipe responsável pela gravação, edição final do documentário e sua possível comercialização.

Para a produção do DVD, alguns integrantes do nosso grupo de pesquisa e alguns profissionais do Laboratório de Análises Clínicas 
da Divisão de Saúde da UFV (LAC/DSA), também cederam sua imagem para as gravações

O público alvo para a confecção desse documentário foram os acadêmicos do curso de graduação em Medicina da UFV, pois os avanços tecnológicos da atualidade têm desencadeado expressivas mudanças no perfil desse público ${ }^{8,9}$, cuja consequência é a necessidade de reorganização do modo de agir do docente, que deve se abrir para a complexidade de métodos alternativos de ensino ${ }^{10-13}$, visando sempre à construção do conhecimento pelos alunos.

A realização desse projeto foi divida em três etapas principais, a preparação, na qual se obteve um embasamento teórico; a execução, onde o DVD foi de fato gravado; e a avaliação final do processo.

\section{Preparação:}

Nessa etapa realizou-se uma busca bibliográfica sobre os procedimentos de coleta de sangue venoso, que consistiu na base para construção dos roteiros de imagem e voz. Além disso, com intuito de fornecer ao grupo de trabalho um maior conhecimento do objeto de estudo pretendido, o recurso audiovisual, foi realizado uma busca sobre o uso dos meios de comunicação como ferramenta de ensino no âmbito da saúde.

Por fim, foi realizada uma busca para verificar a existência de vídeos com a mesma proposta disponíveis na Internet, que pudesse nos orientar no trabalho a ser realizado.

\section{Ехесução:}

As gravações foram realizadas no LAC/DSA. Para realização dessa etapa contamos com a participação dos profissionais do LAC/DSA, de alunos e professores do Departamento de Medicina e Enfermagem (DEM) da UFV, com uma estudante de mestrado do Departamento de Educação Física (DES) da UFV, e a equipe da CEAD.

\section{Avaliação:}

Em concordância com as Diretrizes Curriculares Nacionais do Curso de Graduação em Medicina ${ }^{14}$, nas quais se recomenda $o$ desenvolvimento de competências a partir de vivências reais e contextualizadas da prática, o processo de produção do documentário fundamentou-se no emprego de metodologia ativa, envolvendo todos os atores nas tomadas de decisões ${ }^{15}$, ou seja, visando a troca de conhecimentos entre discentes, docentes e técnicos sobre os conteúdos relativos à coleta e às técnicas de produção de vídeo.

Dessa forma, para uma avaliação qualitativa foram gravados depoimentos de todos os envolvidos na produção do vídeo para análise dos dados de natureza verbal ${ }^{16}$, foram redigidos portfólios por parte dos discentes envolvidos no projeto fazendo relação à perspectiva formativa, tornando o estudante partícipe da própria avaliação ${ }^{17}$ e foram realizadas reuniões em grupo através da técnica de grupo focal para a revisão do processo de ensino-aprendizagem adotado ${ }^{18}$.

A análise final do documentário produzido foi realizada a partir da exibição do mesmo ao público-alvo, sendo avaliado a partir de questionários característicos relacionados à linguagem, tempo de exibição, qualidade da imagem e do som entre outros elementos, buscando cada vez mais melhorar a vertente do ensino da coleta de sangue venoso no âmbito da medicina laboratorial. Os questionários incluíam, também, questões técnicas sobre a coleta de sangue venoso e as variáveis pré-analíticas do processo, cujas respostas constavam no documentário, avaliando o aprendizado desses conteúdos por parte de alunos após assistir o material uma vez.

Os questionários foram aplicados, após exibição do documentário, para estudantes de quatro grupos distintos: alunos de cursos da área de saúde que já haviam estudado coleta de sangue venoso, grupo composto pelas turmas de $2^{\underline{0}}$ e $6^{\circ}$ períodos de Medicina da UFV; alunos da área de saúde que não haviam estudado o tema, composto por alunos do $1^{\circ}$ período de Medicina da UFV e alunos do $7^{0}$ período de Farmácia de uma universidade particular; estudantes de outras áreas, grupo representado por estudantes do $7^{\circ}$ período do curso de Comunicação Social Jornalismo da UFV; e profissionais da área de Análises Clínicas, neste caso funcionários do LAC/DSA. Todos os participantes desta etapa assinaram o Termo de Consentimento Livre e Esclarecido, autorizando a publicação dos resultados dos questionários.

As respostas de cada questão, de acordo com a turma e o grupo, foram somadas e digitalizadas utilizando-se o programa Microsoft Office Excel, com o qual foram realizadas as análises gráficas. 


\section{Resultados}

Os principais resultados obtidos são apresentados de acordo com cada etapa do projeto.

\section{Preparação:}

Além das referências utilizadas ao longo do texto, os procedimentos de coleta foram pautados principalmente nas recomendações da Sociedade Brasileira de Patologia Clínica/Medicina Laboratoria ${ }^{12}$, a partir da qual foi observado que o uso do dispositivo de transferência de amostra sanguínea para coletas com seringa e agulha indicado, não é comum em nossa região, podendo comprometer a qualidade dos exames.

Foi encontrado disponível na Internet um vídeo, dividido em duas partes ${ }^{19,20}$ com uma proposta semelhante à do documentário o qual utilizamos como orientação.

\section{Ехесис̧ão:}

Foram necessários quatro dias de gravação para cumprir o roteiro de voz e imagem planejado e ainda obter os depoimentos de todos que participaram do projeto, sendo obtidos cerca de 60 minutos de imagens do procedimento de coleta de sangue utilizando tubos com vácuo, seringa e punção digital, além de imagens de todos os materiais necessários para o procedimento.

\section{Avaliação:}

A metodologia ativa adotada no processo de produção do documentário, através dos depoimentos, portfólios e do grupo focal, contribuiu para a formação discente tendo estimulado o raciocínio científico, $\mathrm{o}$ aprendizado do trabalho em equipe, a maior interação entre docentes - discentes - técnicos e a integração entre as diferentes áreas de conhecimento, saúde e comunicação.

A técnica do grupo focal se provou de grande valia não apenas para os estudantes que dele participaram, mas reverteu-se em melhora na qualidade do documentário. Os grupos focais foram realizados ao longo do processo de edição do material e a partir destas discussões foram definidas alterações, no sentido de eliminar as falhas no conteúdo apresentado e a sincronizar a elemento audiovisual. A partir desta ferramenta obteve-se a versão final do documentário, sendo a última fase em que ele foi efetivamente modificado.
A análise dos portfólios e dos depoimentos feitos pela equipe que participou da construção desse documentário tem mostrado as dificuldades em lidar com desenvolvimento de um material inovador, mas aponta claramente que todo $\mathrm{o}$ trabalho interdisciplinar foi enriquecedor e que veio a somar conhecimentos. Nos portfólios ficou claro que estar inserido no projeto representava uma experiência nova e que seria um grande desafio criar um material com a qualidade que havia sido proposta. Além disso, a boa relação entre a equipe permitiu superar o nervosismo diante das câmeras e adaptar o ambiente de filmagem, que era inadequado segundo as recomendações bibliográficas.

Já na análise dos depoimentos ficou evidente que a interação entre os profissionais do Laboratório de Análises Clínicas, a equipe do áudio-visual, os professores e os alunos permitiu uma rica troca e construção de conhecimentos. Ficou bem claro que o ensino de coleta de sangue usando imagens, esquemas dinâmicos e voz mostram mais do que simples textos, os quais geram dúvidas frequentes. Como a coleta de sangue é o primeiro procedimento invasivo ensinado no curso médico, o documentário foi visto como um facilitador do ensino dessa prática, a qual é muito útil no dia-a-dia do profissional de saúde.

Outro método de avaliação utilizado foi a aplicação de questionários para 182 pessoas de diferentes grupos. Os questionários foram divididos em três blocos de questões sendo as de 1-5 referentes a opinião pessoal dos espectadores; de 6-10 questões específicas com menor grau de dificuldade; de 11-15 questões específicas com maior grau de dificuldade. Abaixo seguem os resultados globais das questões de 1-5 (Figuras 1, A a D).

Para analisar os resultados obtidos nas questões de 6 a 15 o universo de 182 pessoas foi dividido em subgrupos com características semelhantes. O primeiro subgrupo é constituído da 1 a 3 turma de Medicina da Universidade Federal de Viçosa (UFV) somando, 52 pessoas, as quais todas já haviam aprendido a técnica da punção venosa superficial. O segundo grupo era constituído pelos alunos da quarta turma de Medicina da UFV e pelos que estudavam Farmácia na 
Univiçosa, pois ambos, apesar de serem da área da saúde ainda não haviam aprendido a técnica em questão - somaram 72 pessoas. O terceiro grupo constitui-se de 13 pessoas e inclui o pessoal do Laboratório de Análises Clínicas da UFV e do Laboratório de Habilidades do Departamento de Medicina e Enfermagem da Universidade Federal de Viçosa no qual podemos encontrar técnicos em bioquímica, farmacêuticos e enfermeiros que, entretanto, não tinham contato há tempos com a parte teórica atualizada da técnica de flebotomia. Já o quarto grupo era formado por 35 estudantes do curso de Comunicação Social de 2010 da UFV os quais nunca haviam tido contato com a técnica, mas tinham um grande domínio das técnicas de audiovisual em comparação com os demais. $\mathrm{O}$ maior domínio proporcionou críticas construtivas ao processo de montagem do vídeo que eram escritas, conforme combinado, nas margens do questionário.

Da maneira supracitada obtiveram-se os seguintes resultados (Figuras 2 e 3 ).

\section{Discussão}

O uso de vídeo no ensino tem sido considerado um elemento de atração ou de reforço do interesse do aluno, motivando-o ${ }^{21}$. Isso se dá pela quebra do ritmo que altera a rotina da sala de aula e pela expectativa gerada pela diversificação das atividades ali realizadas. Entretanto, percebe-se que rádio, televisão, vídeo e computador são tecnologias ainda ausentes na escola pública brasileira. Ausentes não no sentido de inexistentes, mas ausentes na utilização, nas discussões e reflexões e, principalmente, na produção ${ }^{18}$. Assim, compreender a importância dessas mídias no contexto educacional é o ponto de partida para a otimização dos recursos disponíveis e complementar o método de ensino até então usado.

A introdução dos recursos audiovisuais nos processos de ensino-aprendizagem foi claramente marcada por um modelo tecnicista, no qual o professor se apoiava em materiais complementares para dar conta de transmitir seu conteúdo com a maior eficácia possível ${ }^{22,23}$. Com a necessidade de acompanhar a tendência atual, incluindo produtos mais preocupados em "entreter os espectadores" sem perder a credibilidade científica das informações transmitidas, vem ocorrendo estudos e mudanças na técnica de produção de documentários, deixando ao longe a idéia uma simples associação de voz, imagem, textos e música de fundo.

Muito se discute sobre a intertextualidade como proposta pedagógica para produção e uso de audiovisual no ensino ${ }^{23}$. A intertextualidade envolve a visão e interpretação dos espectadores sobre aquilo que é apresentado, de forma que as lacunas deixadas no vídeo estimulam a busca e a associação com os conhecimentos prévios, valores e experiências que o aluno já traz no momento da recepção. $\mathrm{O}$ vídeo se torna uma ferramenta para construção conjunta de conhecimentos, onde as informações são articuladas com outras referências, fontes ou textos, façam estes partes ou não da vivência do aluno ${ }^{23}$. Assim, nessa estratégia de ensino é fundamental a intervenção e a fala do professor para direcionar a discussão e dar significado aos conteúdos abordados.

A influência de vídeos educativos na divulgação científica tem mostrado resultados positivos $^{21,24}$, sendo o documentário considerado por alguns autores a mídia preferida pelos jovens para veiculação de determinados temas científicos $^{24}$. O mesmo estudo em que se comparam vídeos de conteúdo mais científico com vídeos de natureza mais leiga sobre o mesmo tema, mostrou que o vídeo de conteúdo mais científico implica aumento de conhecimento do público alvo. Já o vídeo com narrativa mais popularizada, embora tenha uma maior capacidade de atrair a atenção dos telespectadores, não gera impacto significativo no conhecimento científico. Nessa perspectiva, a abordagem científica feita em cada etapa do processo de flebotomia corrobora alguns dados encontrados em nosso trabalho, como a redução nos níveis de dificuldade sobre o tema (Figura 1, D) $\mathrm{e} o$ alto índice geral de acertos do questionário aplicado, após o publico alvo assistir o vídeo (Figura 2).

O documentário foi apresentado ao público alvo, o qual é constituído pelos estudantes e profissionais da área de saúde. O primeiro grupo é composto por estudantes de Medicina e profissionais que já fizeram punção venosa ( $1^{\mathrm{a}}$ e $3^{\mathrm{a}}$ turma de Medicina e profissionais do Laboratório de Análises Clínicas da UFV) e o 

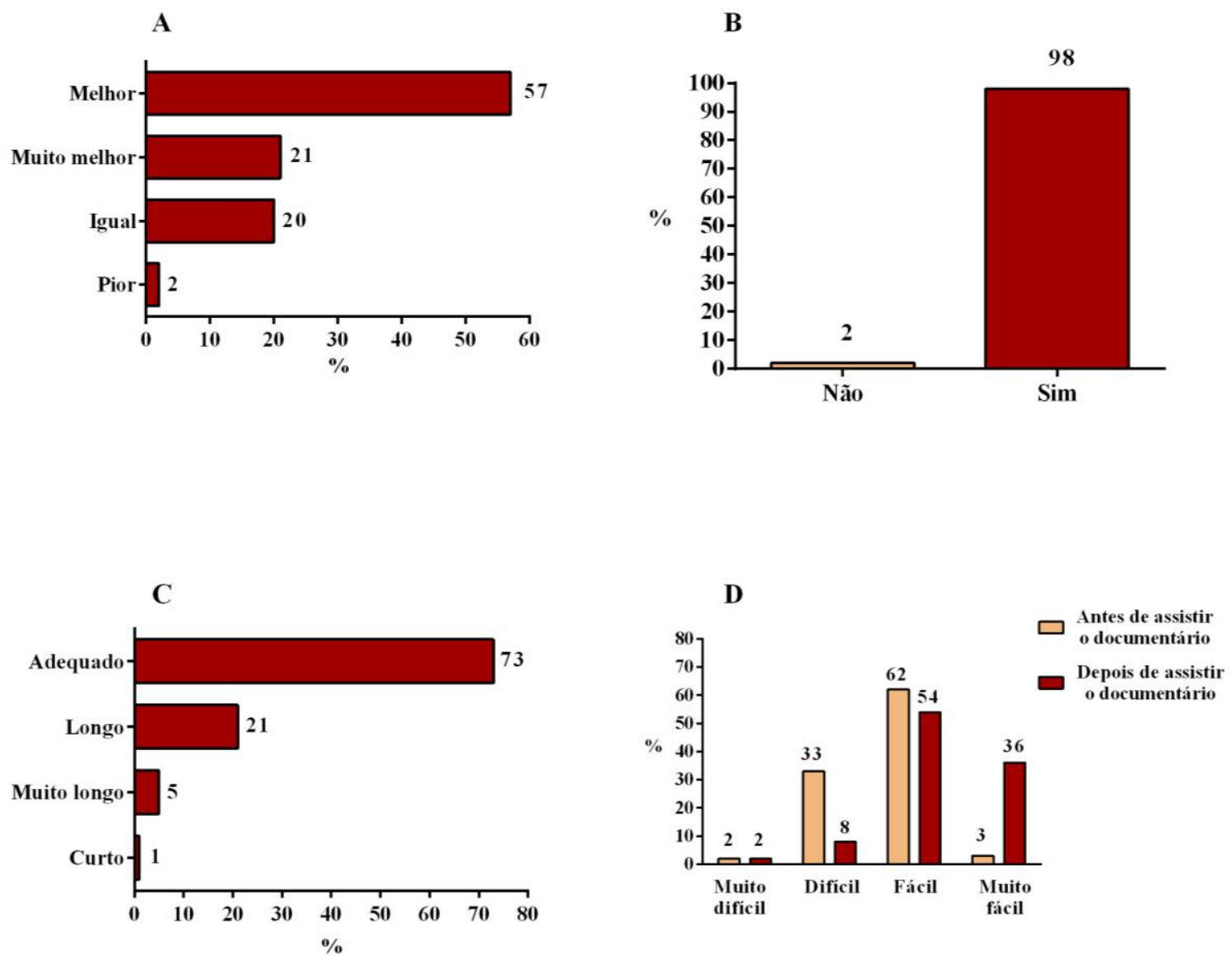

Figura 1. Questões abordadas para avaliação entre o ensino tradicional e a técnica audiovisual. A. Compare o vídeo com ensino tradicional; B. O vídeo foi adequado para seu nível de conhecimento; $\mathrm{C}$. Opinião em relação ao tempo de duração do filme; D. Nível de dificuldade em relação ao conteúdo antes e depois de assistir ao documentário.

segundo grupo inclui estudantes da área de saúde que ainda não tiveram aula sobre coleta de sangue ( $4^{a}$ turma de Medicina e Farmácia). Já o terceiro grupo, diferente dos anteriores, é formado por profissionais da comunicação social, outra área de conhecimento, não relacionada à área da saúde, capaz de avaliar com maior propriedade a qualidade do material elaborado. Em seguida foi aplicado um questionário contendo 15 questões, cujos resultados serão discutidos a seguir.

Dos resultados encontrados, a figura 3 mostra a porcentagem de acertos de dois conjuntos de questões segundo o grupo avaliado. Percebe-se que a porcentagem de acertos das questões de 11 a 15 foram inferiores à porcentagem de acertos das questões de 6 a 10 . O resultado era esperado, uma vez que as primeiras questões, de 6 a 10, eram questões específicas com menor grau de dificuldade e o segundo grupo era composto por questões específicas com maior grau de dificuldade. Além disso, a mesma figura mostra que entre os estudantes da área de saúde, os que já fizeram punção venosa tiverem maior porcentagem de acertos do que aqueles ainda não tiveram aula ou contato com a prática. Já o grupo que não faz parte da área de saúde teve o menor percentual de acertos de todos. Os resultados são coerentes com o perfil diferenciado de cada grupo avaliado.

Na figura 2 mostra a porcentagem de acertos dos grupos avaliados de acordo com as questões de 6 a 15. Nota-se no grupo constituído pelos profissionais do laboratório de análises clínicas um baixo índice de acertos das questões 8 e 14, se comparado aos demais grupos. A questão 8 abordava a higienização das mãos e 


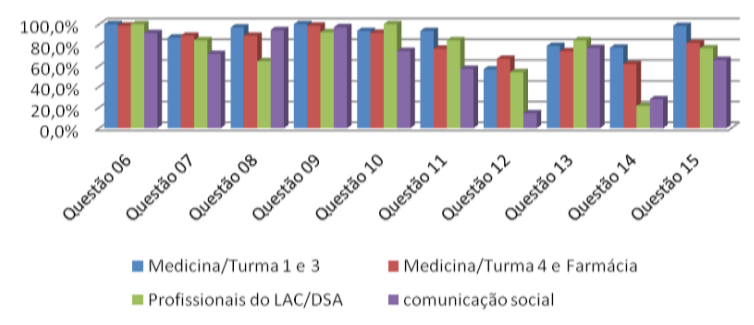

Figura 2 - Porcentagem de acertos dos grupos avaliados de acordo com as questões de 6 a 15 .

antissepsia do local de punção e a questão 14 abordava anatomia e técnica de coleta. Uma justificativa possível para o resultado seria um erro na prática de coleta sanguínea de alguns profissionais do laboratório de analises clínicas, principalmente por esses estarem trabalhando há vários anos e também por haver uma deficiência no processo de reciclagem do conhecimento. Isso reafirma a necessidade da padronização dos procedimentos laboratoriais em um documento (POP) para livre acesso dos funcionários.

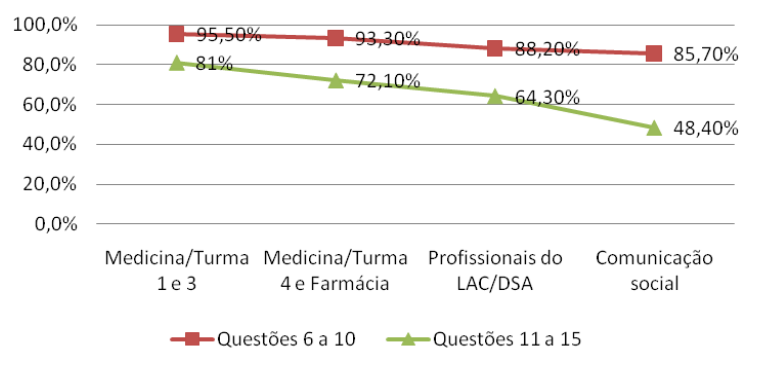

Figura 3 - Porcentagem de acertos segundo o público alvo.

É importante frisar que a diferença de acertos entre cada grupo não foi muito intensa nas questões de menor dificuldade, sendo mais significativa nas questões de maior grau de dificuldade, em que a porcentagem de acertos, de uma forma geral, foi alta. Além disso, ao serem questionados a respeito do conteúdo expresso no vídeo, 98\% responderam que o documentário era adequado para os seus níveis de conhecimento. Disso, percebe-se que o grau de dificuldade em relação ao tema era baixo, o que poderia justificar o grande número de acertos. Em concordância com os dados, na figura $1, \mathrm{D}$ mostras que $62 \%$ dos participantes consideraram o nível de dificuldade do conteúdo, antes de assistir o vídeo, como "fácil". Entretanto, a mesma figura mostra um dado interessante, comparando o nível de dificuldade antes e depois de assistir o documentário: notase uma redução de $33 \%$ para $8 \%$ dos participantes que consideravam o conteúdo como "difícil" e o aumento de $3 \%$ para $36 \%$ dos participantes que consideraram o conteúdo como "muito fácil" após assistirem o documentário. Isso mostra que o documentário atingiu o objetivo de ser um facilitador do processo de ensino-aprendizagem.

No primeiro grupo, composto por estudantes e profissionais que já tiveram contato com a prática de flebotomia percebe-se uma proporção de acertos discrepante. Os estudantes da primeira e terceira turma tiveram os maiores índices gerais de acertos, porém os índices de acertos dos profissionais do laboratório foram inferiores aos índices de acertos dos estudantes que nunca tiveram contato com a técnica de coleta de sangue. As explicações possíveis devem levar em conta as diferenças existentes entre o perfil dos grupos avaliados. Assim, os estudantes por estarem mais familiarizados com os métodos avaliativos do que os técnicos do laboratório e estes, por não sentirem à vontade ao serem avaliados, mesmo que bem orientados sobre a natureza do trabalho, podem ter contribuído para o resultado encontrado. Por outro lado, o maior índice de acerto dos estudantes inexperientes na flebotomia que só tiveram o contato com tema por meio do documentário, em relação os técnicos do laboratório, mostra que os recursos audiovisuais foram úteis no processo de construção do conhecimento dos acadêmicos e funcionam como um bom método de ensino, complementar ao ensino tradicional.

No segundo grupo, representado por acadêmicos que ainda não tinham conhecimento teórico e prático da flebotomia, o percentual de acertos das questões após assistirem o documentário foi alto, diferenciando-se pouco do percentual atingido pelos acadêmicos que já tiveram contato teórico e prático da técnica de punção venosa. Os dados apontam uma equivalência, em termos de eficácia, entre a nova metodologia de ensino e o método de ensino tradicional. No entanto, comprovar a ideia com apenas os dados aqui disponíveis desse grupo são incoerentes com a noção horizontal de 
construção do conhecimento. Os dados foram obtidos a curto prazo e, para se fazer uma análise mais fidedigna dos métodos de ensino, os questionários deveriam ser reaplicados após um certo período de tempo, com o objetivo de avaliar e comparar os dois grupos a longo prazo. Assim, este estudo não mostra a superioridade de um método em relação ao outro, apenas afirma que a metodologia de ensino usando documentário mostrou ser eficaz e um importante instrumento didático para auxílio no ensino.

Já no terceiro grupo, constituído por um público que possui um olhar diferenciado para as questões técnicas e os recursos audiovisuais que envolvem a construção de um documentário, o índice de acertos foi o menor, especialmente nas questões de maior grau de dificuldade. Os resultados eram esperados porque são estudantes de uma área do conhecimento não relacionada à saúde. Entretanto, foram feitas algumas observações sobre as questões técnicas de construção do documentário que precisam ser discutidas aqui.

A primeira é o tempo de duração do vídeo que pareceu longo para o grupo (que representa $20 \%$ do total de participantes). Nesse item, ao se analisar a opinião geral sobre o tempo de duração do vídeo $73 \%$ classificaram-no como "adequado" e apenas 21\% como "longo". Isso mostra que o vídeo foi tido como adequado para o público alvo, provavelmente por questão de afinidade e interesse, cumprindo, então, o seu objetivo. A outra foi uma sugestão de mudança da cor da fonte para criar um contraste maior com o plano de fundo e a última é sobre o fechamento do vídeo que se deu de forma súbita. Como se trata da construção do primeiro documentário, já eram esperados os "erros técnicos" e que os mesmos, não comprometendo a qualidade do conteúdo do vídeo, deveriam apenas ser identificados para não serem repetidos nos próximos documentários. Em suma, o grupo teve uma contribuição para a melhoria da qualidade dos documentários, especialmente os subsequentes.

Percebe-se que uso de documentários no ambiente de ensino é algo promissor. Como pode ser observado na figura 3 , a opinião do público alvo foi favorável ao uso do documentário, tendo-o como um bom método de ensino. Em nossos dados, 78\% dos avaliadores classificaram-no em uma das duas categorias, como "melhor" e "muito melhor" do que o ensino tradicional. Isso mostra a grande aceitação do novo método de ensino e que o uso de recursos audiovisuais permite a criação de ferramentas úteis a serem usadas no âmbito educacional.

De um modo geral, a produção de documentários para ensino de medicina laboratorial representa o início de um estudo sobre uma metodologia até então pouco usada em nosso meio, motivo pelo qual não temos dados sobre seu impacto no ensino como ferramenta rotineira em sala de aula. Assim, este estudo não mostra a superioridade do novo método de ensino em relação ao tradicional, apenas afirma que a nova metodologia usando documentário mostrou ser eficaz e um importante instrumento didático para auxílio do método tradicional de ensino, sendo o professor um mediador de todo o processo. Como há pouca pesquisa sobre o desenvolvimento de materiais audiovisuais, estudos adicionais são necessários para oferecer elementos para a construção de materiais educativos que superem o modelo tradicional.

\section{Referências}

1. Campana GA, Oplustil CP, de Faro LB. Tendências em medicina laboratorial. J Bras Patol Med Lab 2011; 47(4):399-408.

2. Sociedade Brasileira de Patologia Clínica/Medicina Laboratorial (SBPC/ML). Recomendações da Sociedade Brasileira de Patologia Clínica/Medicina Laboratorial para a coleta de sangue venoso. 2 ed. Barueri: Minha Editora; 2010.

3. Lima-Oliveira GS, Picheth G, Sumita NM, Scartezine M. Controle da qualidade na coleta do espécime diagnóstico sanguíneo: iluminando uma fase escura de erros préanalíticos. J Bras Patol Med Lab 2009; 45(6):441-7.

4. Messeder JC, Rôças G. O Lúdico e o ensino de ciências: um relato de caso de uma licenciatura em química. Rev Ciênc \& Ideias 2009-2010; 1(1):69-5.

5. Napolitano M. Como usar o cinema na sala de aula. São Paulo: Contexto; 2006. 
6. Mori S, Whitaker IY, Marin HF. Estratégias tecnológicas de ensino associadas ao treinamento em Suporte Básico de Vida. Acta Paul Enferm 2011; 24(5):721-5.

7. Siqueira-Batista R, Gomes A, Rôças G, Leite S. $O$ cinema na formação bioética de professores de ciências. Anais do Encontro Nacional de Ensino de Ciências da Saúde e do Ambiente Niterói: UNIPLI. 2008: 309-17.

8. Aguilar-da-Silva RH, Perim G, Abdala I, Costa N, Lampert J, Stella R. Abordagens pedagógicas e tendências de mudanças nas escolas médicas. Rev Bras Educ Med 2009; 33(1 supl 1):53-62.

9. WHO. Changing medical education and practice: an agenda for action. Genebra; 1991.

10. Ousager J, Johannessen H. Humanities in undergraduate medical education: A literature review. Acad Med 2010; 85(6):988.

11. Buckley S, Coleman J, Davison I, Khan KS, Zamora J, Malick S, et al. The educational effects of portfolios on undergraduate student learning: a Best Evidence Medical Education (BEME) systematic review. BEME Guide No. 11. Med Teach 2009; 31(4):282-98.

12. Steinert $\mathrm{Y}$, Mann K, Centeno A, Dolmans D, Spencer J, Gelula $M$, et al. A systematic review of faculty development initiatives designed to improve teaching effectiveness in medical education: BEME Guide No. 8. Med Teach 2006; 28(6):497-26.

13. Colthart I, Bagnall G, Evans A, Allbutt H, Haig A, Illing J, et al. The effectiveness of selfassessment on the identification of learner needs, learner activity, and impact on clinical practice: BEME Guide no. 10. Med Teach. 2008; 30(2):124-45.

14. CNE/CES. BCSRN. Diretrizes Curriculares Nacionais do Curso de Graduação em Medicina. Brasília: Diário Oficial da União; 2001. p. 38.

15. Pedrosa IL, Lira GA, Oliveira B, Silva MSML, Santos MB, Silva EA, et al. Uso de metodologias ativas na formação técnica do agente comunitário de saúde. Trab Educ Saúde 2011; 9(2):319-32.

16. Lefevre AMC, Crestana MF, Cornetta VK. A utilização da metododologia do discurso do sujeito coletivo na avaliação qualitativa dos cursos de especialização "Capacitação e Desenvolvimento de Recursos Humanos em Saúde-CADRHU”, São Paulo - 2002. Saúde Soc 2003; 12(2):68-5.

17. Gomes AP, Arcuri MB, Cristel EC, Ribeiro RM, Souza LMBM, Siqueira-Batista R. Avaliação no Ensino Médico: o Papel do Portfólio nos Currículos Baseados em Metodologias Ativas. Rev Bras Educ Med 2010; 34(3):390-6.

18. Iervolino SA, Pelicione MCF. A utilização do grupo focal como metodologia qualitativa na promoção da saúde. Rev Esc Enf USP. 2001; 35(2):115-21.

19. Ministério da Saúde, Programa Nacional de Doenças Sexualmente Transmissíveis e Aids dMdS. [Vídeo], Técnicas para coleta de sangue Parte 1/2; [9 min., 2 s.]. Disponível em: https://www.youtube.com/watch?v=TqiEQrn $\underline{B N K Y}$. Acesso em março de 2012.

20. Ministério da Saúde, Programa Nacional de Doenças Sexualmente Transmissíveis e Aids dMdS. [Vídeo], Técnicas para coleta de sangue Parte 2/2; [8 min., 8 s.]. Disponível em: https://www.youtube.com/watch?v=6CpgQ9j IPGs . Acesso em março de 2012.

21. Junior AAP, Rezende LAC, Bastos WG. Recepção Audiovisual na Educação Médica: Leituras de um Vídeo Educativo de Psicologia Médica por Estudantes de Medicina. Rev Bras Educ Med 2012; 36(4):51623.

22. Souza SMO. Tiza, cámara, acción: la imagen en la formación de educadores. Comunicar 31(16): 485-490; 2008.

23. Rezende LA, Struchiner M. Uma Proposta Pedagógica para Produção e Utilização de Materiais Audiovisuais no Ensino de Ciências: análise de um vídeo sobre entomologia. Alexandria Rev. Educ Ciênc Tecnol 2009; 2(1):45-66.

24. Camargo BV, Barbará A, Bertoldo RB. A influência de vídeos documentários na divulgação Científica de conhecimento sobre a AIDS. Psicol Refl Crít 21(2), 179-185. 2007. 